\title{
Histopathological Image Classification Using Stain Component Features on a pLSA Model
}

\author{
Gloria Díaz and Eduardo Romero* \\ Bioingenium Research Group, National University of Colombia, \\ Bogotá, Colombia \\ \{gmdiazc, edromero\}@unal.edu.co
}

\begin{abstract}
Semantic annotation of microscopical field of views is one of the key problems in computer assistance of histopathological images. In this paper a new method for extracting patch descriptors is proposed and evaluated using a probabilistic latent semantic analysis (pLSA) classification model. The proposed approach is based on the analysis of the different dyes used to stain the histological sample. This analysis allows to find local regions that correspond to cells in the image, which are then described by the SIFT descriptors of the stain components. The proposed approach outperforms the conventional sampling and description strategies, proposed in the literature.
\end{abstract}

Keywords: Semantic annotation, Histopathological Images, Color decomposition, pLSA, SIFT descriptors.

\section{Introduction}

Computer-aided diagnosis (CAD) for histopathology images is an emerging research field, which has become popular because of the recent advances in capturing devices as well as in the increasing computational capacities that not only have facilitated digitization, storing and distribution of microscopic samples, but also has allowed the development of image analysis tools that support diagnosis, teaching and research processes [1]. In particular, computer aided image analysis can help pathologists to identify suspicious areas so that they can dedicate their analysis time to these areas, whereby their workload can be highly reduced and therefore the response delays of health systems.

One main challenge when developing histopathology CADs consists in building an effective model for extracting information that allows to determine the semantic meaning from the visual content of the image. This task has been twofold approached as a classification problem: either as classes assigned to the whole image, according to global descriptors, or as classes assigned to histological structures previously segmented [2]. In the former case, global descriptors are hardly able to extract relevant visual features from specific cytological components, a key factor

\footnotetext{
* This work was supported by the Colombian Administrative Department of Science and Technology (COLCIENCIAS).
} 
for the semantic description, while in the latter case the classification performance is straightforwardly related to the segmentation accuracy, a very difficult job when one considers the complexity of histopathological images.

Recently, patch-based representation schemes and probabilistic hidden models have shown excellent performance for unsupervised semantic description of images [3]. Specifically, the probabilistic latent semantic analysis (pLSA) [4], a generative model which tries to learn latent concepts or topics from a bagof-features (BOF). Main challenges in such representations are extraction of the most discriminative patches and descriptors, which capture main statistical properties and therefore semantic categorization approaches can be improved. Caicedo et al. evaluated the BOF representation in histopathological images [5]. Interestingly, they found that this representation can be related to semantic concepts in histopathology images, but its performance is dependent on both the region selection and description.

In this paper a new method for extracting patch descriptors in histopathological images is proposed and evaluated on a pLSA classification model. This approach takes advantage of a specific image characteristic, obtained from histopathological slides, which are stained with Hematoxylin-Eosin. Hematoxylin stains cell nuclei in blue-purple, while Eosin stains cytoplasm and connective tissue in pink. These colors constitute the base upon which pathologists are able to distinguish cellular tissue components. Likewise, we used these properties for finding distinctive image markers that correspond to cell nuclei, and generate discriminative descriptors that amount to the stain absorbed by tissues.

\section{Latent Topics Annotation Model}

\section{1 pLSA model}

Probabilistic Latent Semantic Analysis (pLSA) is a generative model, which probabilistically describes how words, in a document, might be generated using latent variables [4. This model assumes that a document $d$ and a word $w$ are conditionally independent given the unobserved topic $z$, by which each document $d_{i}$ is a mixture of latent topics, a process modeled by a multinomial distribution $P\left(z \mid d_{i}\right)$, and each latent topic $z_{l}$ is also modeled by an additional multinomial distribution $P\left(w \mid z_{l}\right)$. The process of generating the set of observations $(w, d)$ can be described by the probabilistic model defined by eq. 1 .

$$
P\left(d_{i}, w_{j}\right)=P\left(d_{i}\right) P\left(w_{j} \mid d_{i}\right), \quad P\left(w_{j} \mid d_{i}\right)=\sum_{l=1}^{k} P\left(w_{j} \mid z_{l}\right) P\left(z_{l} \mid d_{i}\right)
$$

As the topic distribution is not an observed variable, the unobservable probability distribution $P\left(z_{l} \mid d_{i}\right)$ and $P\left(w_{j} \mid z_{l}\right)$ can be learned from the likelihood of the observed data given by the eq. 2 .

$$
L=\prod_{i=1}^{M} \prod_{j=1}^{N} P\left(w_{i} \mid d_{j}\right)^{n\left(w_{i}, d_{j}\right)}
$$


where $N$ is the number of documents, $M$ is the number of words in the vocabulary, $n\left(w_{i}, d_{j}\right)$ is the number of occurrences of a word $w_{i}$ in document $d_{j}$ and $P\left(w_{i} \mid d_{j}\right)$ is given by eq. 11. Best model parameters are found using the Expectation Maximization (EM) algorithm, which iteratively estimate the posterior probabilities for the latent variables $P\left(z_{k} \mid d_{i}, w_{j}\right)$ (expectation step) and optimize this estimation $P\left(w_{j} \mid z_{k}\right)$ and $P\left(z_{k} \mid d_{i}\right)$ (maximization step) until convergence.

The latent concept probabilities, from the observed words, are estimated for a new document $d_{\text {test }}$, using a partial version of the EM algorithm described previously, but the conditional probability distribution $P\left(w_{j} \mid z_{k}\right)$ is kept fixed i.e. this is not updated at each M-step.

\subsection{Representing Images as Bag of Words}

The pLSA model represents documents as a set of words. From an image analysis standpoint, the image semantic may be captured when the global scene is expressed in terms of its components. The Bag-of-Features representation aims to follow this principle when documents amount to whole images and words correspond to quantized local image descriptors named visual words [6]. The BoF is generated by selecting a set of local image regions, which are characterized in a feature space. Then, instances in the feature space are grouped using a conventional clustering algorithm (e.g. k-means), defining a fixed number of groups that corresponds to words in the visual vocabulary. K-clusters are finally used for representing the image by a histogram of visual words, resulting from assigning each local region to the nearest k-cluster center.

\subsection{Image Annotation Based on the pLSA Model}

Once the posterior probability distribution of concepts $P\left(z \mid d_{i}\right)$ is computed, image annotation can be twofold performed: 1)if images can be labeled with a unique concept, the number of latent concepts is set to the number of class labels, and the annotation process consists in finding the concept that has higher conditional probability $P\left(z \mid d_{i}\right)$. This case requires that image concepts are highly correlated, and that there exists only a single type of background, specific for each class label. 2)if image can not be uniquely labeled, the number of concepts can be different of the number of class labels, and the concept probability distribution is used as a feature vector (eq. 3) for training a discriminative classification model. In this work the latter approach was evaluated using the $\mathrm{k}$-nn rule as classification model.

$$
d_{i}=\left\{P\left(z_{1} \mid d_{i}\right), P\left(z_{2} \mid d_{i}\right), \ldots, P\left(z_{k} \mid d_{i}\right)\right\}
$$

\section{Local Region Description Based on the Staining Component Analysis}

Histopathological concepts are mainly characterized by cells that are visually identified by their color, which result from the affinity of tissue components to 
particular dyes. Our aim was to find the most discriminative local regions for the classification task. For so doing, we propose to find the cell nuclei and describe the regions around them. The extraction of local regional descriptors consists in: color normalization, cell based local region detection and local region description of the staining components.

\subsection{Color Normalization}

The obserbed color at each pixel is proportional to the strength of a chemical reaction between a dye and the biological substances. Many factors such as environment illumination conditions, relative dye quantity or film inhomogeneities produced by the subsequent slide storage and handling, result in a high luminance and color variability, wich strongly affects the staining component based analysis. This problem was solved by applying the color normalization approach proposed by Reinhard et al. [7].

\subsection{Local Region Selection}

Decomposing the image in main stain factors. The main goal was to accurately separating the image into the colors that corresponded to the actual contribution of each stain (Hematoxylin-eosin). For doing so, it was assumed that each RGB color can be described as a linear combination of the two stain factors. Deconvolution process consisted on separating the two stain contributions as described in eq. 4, under the physical restriction that negative stain contribution were not possible. So, $D$ and $S$ were found using the non-negative matrix factorization method [8]. Images were converted to optical density (OD) values before than deconvolution process was applied.

$$
\left[\begin{array}{l}
R \\
G \\
B
\end{array}\right]=\left[\begin{array}{ll}
D 1_{1} & D 2_{1} \\
D 1_{2} & D 2_{2} \\
D 1_{3} & D 2_{3}
\end{array}\right]\left[\begin{array}{ll}
S_{1} & S_{2}
\end{array}\right]
$$

where $D 1, D 2$ correspond to the two stain vectors, and $S_{1}, S_{2}$ indicate the stain contribution. Figure 1 show results from deconvolution process of selected images. First row, corresponds to input images, second row corresponds to the first stain component, which show the eosin dye contribution at each pixel. Finally, the hematoxylin dye contribution is drawn in the third row.

Extracting visual patches. Once the stain contribution was determined, the cell nuclei were detected on the Hematoxylin component using a simple Otsu threshold, followed by a morphological grey-scale opening filter using a $2 \times 2$ structuring element. On the other hand, the clumped cells were split as much as possible applying a morphological grey-scale closing filter on the segmented regions with areas larger than 100 pixels. Finally, circular patches were extracted around the centre of the detected nuclei, as shown in the four row of Figure 1. 

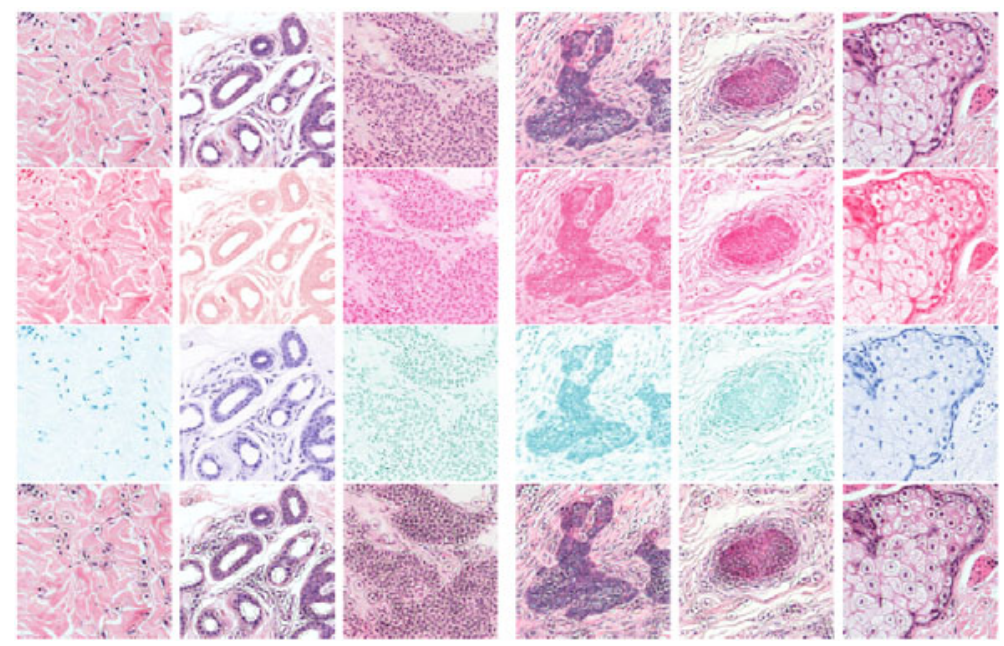

Fig. 1. Stain decomposition of selected samples from the training dataset

\subsection{Local Region Description}

Local patches were represented using the SIFT descriptor proposed by Lowe [9], which describes the texture of local regions, using edge orientation histograms. Traditionally, SIFT descriptor is computed under light intensity changes. However, color information is critical in histopathological image analysis. So, the use of color information inside the SIFT descriptors was evaluated. Images were decomposed in representative color spaces (rgSIFT, cSIFT and stnSIFT), and the SIFT descriptor for each color component was computed using a common parameter configuration i.e. $4 \times 4$ blocks and 8 orientations, resulting in a 128-dimensional feature vector for each color component.

- rgSIFT descriptor that results from the concatenation of SIFT descriptors computed under $r$ and $g$ chromaticity components of the normalized RGB color space (eq. 4).

$$
r_{n}=\frac{R}{R+G+B} \quad g_{n}=\frac{G}{R+G+B}
$$

- cSIFT descriptor, corresponding to the concatenation of SIFT descriptors computed under the two components of the normalized opponent color space.

$$
\begin{gathered}
C_{1}=\frac{C_{1}}{C_{3}}, C_{2}=\frac{C_{2}}{C_{3}} \\
\text { with, } C_{1}=\frac{R-G}{\sqrt{2}}, \quad C_{2}=\frac{R+G-2 B}{\sqrt{6}, \quad C_{3}=\frac{R+G+B}{\sqrt{3}}}
\end{gathered}
$$

- stnSIFT descriptor, corresponding to the concatenation of SIFT descriptors computed under the two stain channels obtained from the deconvolution process. 


\subsection{Model Evaluation}

The proposed local region detection and description methods were compared with traditional grid based and sift point detection sampling strategies 10. In the former case images were split in a regular grid of $21 \times 21$ pixels per block. In the latter, SIFT points were detected according to Lowe 9 . In both cases, circular local regions defined in a radius of 10 pixels were described as explained in the section 3.3 and processed by the pLSA model.

Images represented by the posterior probability distribution of hidden topics $P\left(z \mid d_{i}\right)$ were classified using the traditional $K-n n$ learning model. Vocabulary size, number of topics and $k$ parameters of the learning model were evaluated. Performance of the classification tasks was quantified in terms of effectiveness measure $F_{\beta}$ computed as $F_{\beta}=\frac{2 * P R * R C}{P R+R C}$, with $P R$ and $R C$ the well known precision and recall performance measures.

\section{Experiments and Results}

\subsection{Data Set}

A total of 540 images acquired from histopathological skyn biopsies, stained with Hematoxylin-Eosin, were used. Each was annotated by experts, with one from nine possible class labels, resulting in 54 Pilosebaceous anexa (PA),71 Nodular, 62 basal cell carcinoma (NBC), 62 Micro-nodular basal cell carcinoma (MnBC), 51 Morpheiphorm basal cell carcinoma (MBC), 68 Epidermis (EP), 60 Sebaceous glands (SG), 51 Eccrine glands (EG), 58 Lymphocyte infiltration (LI) and 76 Collagen (CO) images. The dataset was randomly divided into training $(80 \%)$ and test (30\%) image sets. Images were cropped for containing only one of foreground concepts.

\subsection{Results and Discussion}

The first evaluation focused on the markers detection stage. Local regions extracted with the proposed approach, with the sift point detector and with the regular grid partition, were used for constructing a visual vocabulary that was input to the pLSA analysis. Local regions were described using the conventional SIFT descriptor. Although different vocabulary sizes were evaluated $(50,100$, 200, 300 and 500) we report results obtained with 300 clusters, because they produced the best average performance for each parameter set (results not shown). Figure 2-(a) shows a plot of $F_{\beta}$ measure reported per each sampling strategy with $k=9$, for the different number of topics. The proposed point detection strategy reports better performance that points obtained from regular grid partition and SIFT detector in about 20\%. Interestingtly, in this experiment the number of topics is not critical for performance, i.e. standar deviation reported was 0.06 .

The second evaluated factor was the effect of using color information in the region descriptor. Figures 2 - (b-d) show plots of $F_{\beta}$ measures reported by varying the region description in the three sampling strategies: SIFT detector in 

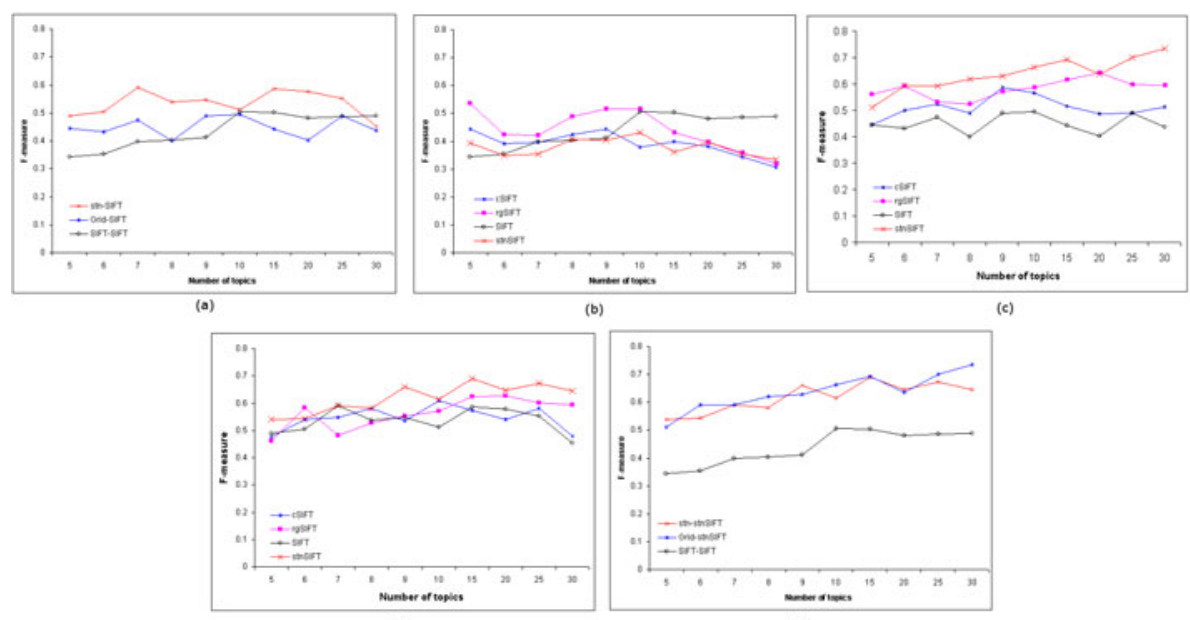

(d)

(e)

Fig. 2. Classification results in the test set (view text for explanation)

2.(b), regular grid partition in 2 (C) and the proposed approach in 2 (d). In these experiments the number of clusters and $k$ parameter were set to 300 and 9, respectively. As expected, the results show that description computed from the illumination channel has in general poorer performance with respect to the color based description, except when regions are located around SIFT detected points. In that case, the color information effect is not clear, although rgSIFT and CSIFT descriptors report better performance than the illumination channel, when the number of concepts is smaller than the number of class labels. This bias changes when the number of topics increases, probabilly because SIFT points were detected under the illumination channel, whereby this component's contribution, for describing these points, is larger. On the other hand, in both regular grid partition and the proposed region selection, the color contribution is remarkable. Furthermore, the description based on the tissue components, defined by the staining contribution, outperforms the other color based descriptors with a maximal $F_{\beta}$ measure of 0.73 for the regular grid partition and 0.69 for the staining based detected regions. Finally, Figure 2-(e) summarizes the results, plotting the best performance obtained for each point detection-descriptor combination. The black line, the poorest performance, corresponds to the results with regions obtained by conventional SIFT detector and descriptor. The red line corresponds to the results obtained by the proposed approach and they are comparable with those obtained from a regular grid partition described by the staining composition (blue line). However, the proposed approach is more efficient in terms of extraction of features and learning of the model, because a smaller number of regions is sampled, especially for images with a very small number of cells. 


\section{Conclusions}

This paper presented a new strategy for selecting and describing local regions for improving the classification performance of histopathological images in a probabilistic latent semantic analysis framework. The proposed approach was based on the decomposition of histological images in their main stain contributions, which ideally allows to describe the biological tissue components. Image classification, based on the proposed approach, outperforms the conventional methods as regular grid partition and SIFT point detector. Moreover, describing local regions by measurement of stain components increased the classification performance based on the regular grid partition doing that comparable with our results.

\section{Acknowledgments}

Histopathology slides used in this study were supplied by the Pathology Department of the National University of Colombia.

\section{References}

1. Romero, E., Gomez, F., Iregui, M.: Virtual Microscopy in Medical Images: a Survey. In: Modern Research and Educational Topics in Microscopy. Formatex (2007)

2. Gurcan, M., Boucheron, L., Can, A., Madabhushi, A., Rajpoot, N., Yener, B.: Histopathological image analysis: A review. IEEE Reviews in Biomedical Engineering 2, 147-171 (2009)

3. André, B., Vercauteren, T., Perchant, A., Buchner, A.M., Wallace, M.B., Ayache, N.: Endomicroscopic image retrieval and classification using invariant visual features. In: Proceedings of International Symposium on Biomedical Imaging (2009)

4. Hofmann, T.: Unsupervised learning by probabilistic latent semantic analysis. Machine Learning 42, 177-196 (2001)

5. Caicedo, J.C., Cruz-Roa, A., Gonzalez, F.A.: Histopathology image classification using bag of features and kernel functions. In: Combi, C., Shahar, Y., Abu-Hanna, A. (eds.) AIME 2009. LNCS, vol. 5651, pp. 126-135. Springer, Heidelberg (2009)

6. Sivic, J., Zisserman, A.: Video google: A text retrieval approach to object matching in videos. In: International Conference on Computer Vision (2003)

7. Reinhard, E., Ashikhmin, M., Gooch, B., Shirley, P.: Color transfer between images. IEEE Transactions on Computer Graphics and Applications 21, 34-41 (2001)

8. Lee, D.D., Seung, H.S.: Algorithms for nonnegative matrix factorization. Advances in Neural Information Processing Systems 13, 556-562 (2001)

9. Lowe, D.: Distinctive image features from scale-invariant keypoints. International Journal of Computer Vision 60, 91-110 (2004)

10. Nowak, E., Jurie, F., Triggs, B.: Sampling strategies for bag-of-features image classification. In: Leonardis, A., Bischof, H., Pinz, A. (eds.) ECCV 2006. LNCS, vol. 3954, pp. 490-503. Springer, Heidelberg (2006) 